Leitthema

Pneumologe 2021 · 18:212-217

https://doi.org/10.1007/s10405-021-00385-2

Angenommen: 3. Februar 2021

Online publiziert: 5. März 2021

(c) Springer Medizin Verlag GmbH, ein Teil von

Springer Nature 2021

\section{Redaktion}

C. Vogelmeier, Marburg

B. Schmeck, Marburg

Die Pneumonie wird in der Regel durch eine akute Infektion der unteren Atemwege hervorgerufen. Sie führt weltweit jährlich zum Tod von etwa 3 Mio. Menschen und verursacht eine hohe Zahl von Krankenhauseinweisung und Antibiotikabehandlungen [1]. In den letzten Jahren wurde offensichtlich, dass auch die Langzeitmortalität durch Lungenentzündungen erhöht wird, unter anderem aufgrund vermehrter kardiovaskulärer Ereignisse [2]. Die häufigsten Erreger der Pneumonie sind Bakterien wie Streptococcus pneumoniae und Haemophilus influenzae, aber auch intrazelluläre Mikroben wie Legionella pneumophila. Die jährlichen Influenzaepidemien verursachen ebenfalls eine hohe Morbidität und Mortalität. Coronaviren haben bisher 3 große Ausbruchgeschehen hervorgerufen: die SARS-CoV-1-Pandemie 2002, den MERS-Ausbruch 2013 und die aktuelle SARS-CoV-2-Pandemie mit bisher über 102 Mio. Erkrankten und über 2,2 Mio. Toten weltweit [3].

Zur Einschätzung der Prognose und zur Wahl der kalkulierten Therapie werden v. a. der Ort und die Umstände der Infektion (ambulant-erworben, nosokomial, beatmungsassoziiert) und klinische Eigenschaften der Patienten (Alter, Vorerkrankungen, Medikation) herangezogen. Andererseits gibt es schon lange die Beobachtung, dass zwar viele Menschen einem Infektionsrisiko ausgesetzt sind, jedoch nur wenige erkranken [4]. Vor der Identifikation von Mycobacterium tuberculosis wurde die Tuberkulose teilweise als erbliche Erkrankung angesehen, da sie häufig in Familien auftrat. Aktuelle Studien bestätigen zuneh-

\author{
Wilhelm Bertrams ${ }^{1}$ Anna Lena Jung ${ }^{1}$ Michael Maxheim ${ }^{2} \cdot$ Bernd Schmeck $^{1,2}$ \\ 'Institut für Lungenforschung, Universities of Gießen and Marburg Lung Center (UGMLC), Philipps- \\ Universität Marburg, Marburg, Deutschland \\ ${ }^{2}$ Klinik für Innere Medizin mit Schwerpunkt Pneumologie, Universitätsklinikum Marburg, Philipps- \\ Universität Marburg, Marburg, Deutschland
}

\title{
Bedeutung der Genetik für die Pneumonie und COVID-19
}

mend, dass die individuelle Suszeptibilität für Infektionskrankheiten im Allgemeinen und die Pneumonie im Besonderen in der Tat auch eine genetische Grundlage hat. In einer dänischen Kohortenstudie wurden 960 Familien mit nicht verwandten Adoptivkindern in Bezug auf vorzeitige Todesfälle beobachtet [5]. Wenn die biologischen Eltern der Kinder vorzeitig an einer Infektionskrankheit verstarben, erhöhte sich das relative Risiko der Kinder, ebenfalls an einer Infektionskrankheit zu versterben, auf das 5,8-Fache, während ein vorzeitiger Tod durch Infektionskrankheiten der Adoptiveltern keinen Einfluss hatte. Diese Erhöhung des relativen Risikos war höher als etwa für kardiovaskuläre Erkrankungen oder Krebs. Das vermehrte Auftreten schwerer bakterieller Infektionen erfordert also immer auch die Betrachtung einer möglichen genetischen Ursache.

\section{Primäre monogenetische Immundefekte}

Grundlage einer erhöhten Infektanfälligkeit können primäre monogenetische Immundefekte sein, von denen bereits über 300 beschrieben wurden. Sie sind in der Regel selten, zeigen aber einen starken Phänotyp, häufig in Bezug auf ein oder wenige Pathogene [6].

Ein typischer monogenetischer Immundefekt ist die 1952 beschriebene Bruton-Agammaglobulinämie. Diese X-chromosomal-rezessive Erkrankung geht mit multiplen invasiven Pneumokokkeninfektionen und anderen pyogenen bakteriellen Infekten einher.
Durch die aktuellen Sequenzierungen des Exoms oder des gesamten Genoms werden immer weitere Einzelnukleotidmutationen (SNPs) in Immungenen identifiziert [7]. Jedoch zeigen gerade die Mechanismen der angeborenen Immunität eine hohe Redundanz, sodass der beobachtete Phänotyp als Konsequenz solcher SNPs häufig gering ist.

In genetischen Untersuchungen einer Gruppe von Kindern mit wiederkehrenden invasiven Pneumokokkeninfektionen wurde die zentrale Rolle eines Signalwegs der angeborenen Immunität für die Infektion mit bekapselten Bakterien aufgezeigt [4]: Diese Bakterien werden über membranständige Rezeptoren, sog. Toll-like-Rezeptoren (TLRs), erkannt, die über mehrere Signalmoleküle zur Aktivierung des Transkriptionsfaktors NF- $\kappa B$ führen, der eine Vielzahl proinflammatorischer $\mathrm{Me}$ diatoren und Effektoren reguliert. Autosomal-rezessiv vererbte Mutationen in den Signalmolekülen MYD88 und IRAK4 führen $\mathrm{zu}$ schweren, invasiven Infektionen mit pyogenen bekapselten Bakterien bei Kindern. Die Abwehr gegen andere Bakterien, Mykobakterien, Viren und Pilze ist kaum gestört. Mutationen in den Genen IKBKG und NFKBIA beeinträchtigen jedoch auch die Immunabwehr gegen atypische Mykobakterien, Viren und Pilze. Die Infektionshäufigkeit nimmt mit dem Alter ab. Am Beispiel vermehrter Staphylococcusaureus-Infektionen durch eine TIRAPMutation konnte dies auf das Auftreten schützender Antikörper zurückgeführt werden. Das heterozygote Auftreten solcher Mutationen kann aber durch 
Tab. 1 Genetische Risikofaktoren für einen schweren Verlauf von COVID-19. (Angepasst aus [23]) Risikofaktor Risikoschätzung Allelfrequenz

Populationsbasierte genetisch epidemiologische Studien (häufige Varianten)

Cluster mit intronischen Varianten von SLC6A20, LZTFL1, 2,11 CCR9, FYCO1, CXCR6 und XCR1

Cluster mit intronischen Varianten von OAS1, OAS2 und 1, OAS3

Cluster mit intronischer Variante von DPP9 1,36

Cluster mit intronischer Variante von IFNAR2 1,28

Patientenbasierte genetische Studien (seltene Varianten)

TLR3, UNC93B1, TICAM1, TBK1, IRF3, IRF7, IFNAR1, IFNAR2 (autosomal-dominant)

IRF7, IFNAR1 (autosomal-rezessiv)
$0,08(0,001-0,28)$

$0,64(0,50-0,78)$

$0,33(0,13-0,41)$

$0,71(0,40-0,78)$

$<0,001$

$<0,001$ eine Dämpfung der Immunabwehr auch $\mathrm{zu}$ einem protektiven Effekt gegen die Entwicklung einer Sepsis führen. In Kandidatengenstudien wurden auch für Legionella pneumophila, dem häufigsten intrazellulären Erreger der Pneumonie, Hinweise auf eine erhöhte Suszeptibilität durch SNPs in Signalwegen der Pathogenerkennung (TLR5, TLR6, STING, TOLLIP) identifiziert [8-11].

Schwer verlaufende Influenzapneumonien wurden mit autosomal-rezessiv vererbten SNPs in Verbindung gebracht [7]. In diesen Fällen wurde v. a. die Produktion oder Wirkung von Typ-Ioder Typ-III-Interferonen durch einzelne Basenaustausche in den Genen IRF7, IRF9 und TLR3 beeinträchtigt.

》) Schwer verlaufende Influenzapneumonien wurden mit autosomal-rezessiv vererbten SNPs in Verbindung gebracht

Ein weiterer monogenetischer Immundefekt ist die mendelsche Anfälligkeit für Erkrankungen durch Mykobakterien (MSMD) [4]. Initial wurde eine seltene mendelsche Vererbung einer erhöhten Empfindlichkeit gegenüber atypischen Mykobakteriosen und Salmonelleninfektionen durch 31 verschiedene autosomal-heterozygot vererbte Mutationen in 16 Genen im Interleukin-12/Interferon$\gamma$-Signalweg beobachtet. Kürzlich zeigte sich jedoch eine unvollständige Penetranz, sodass bei einigen homozygoten Genträgern als einziger Phänotyp eine erhöhte Anfälligkeit gegenüber Infek- tionen mit Mycobacterium tuberculosis auftrat. Eine neue Studie legt nahe, dass die TYK2(P1104A)-Mutation für 1\% aller Tuberkulosefälle bei Patienten mit europäischer Abstammung verantwortlich ist [12].

Darüber hinaus wird die Immunantwort der Lunge auch durch die genetischen Erkrankungen der zystischen Fibrose und des a1-Antitrypsin-Mangels beeinträchtigt, die an anderer Stelle diskutiert werden.

\section{Erhöhte Suszeptibilität durch Genpolymorphismen}

Das humane Genomprojekt und die Verfügbarkeit von Microarrays zur Analyse einer Vielzahl von SNPs haben es ermöglicht, durch genomweite Assoziationsstudien (GWAS) eine Vielzahl von krankheitsassoziierten Genvarianten zu identifizieren, die für sich genommen nur einen kleinen Risikozuwachs (1,1- bis 1,5-fach) bewirken.

\section{》) Durch GWAS konnte eine Vielzahl von krankheitsassozi- ierten Genvarianten identifiziert werden}

Die größte dieser Studien wurde an der Vanderbilt-University durchgeführt [13]: Bei 69.819 Personen mit europäischem Hintergrund wurden 8889 Pneumonien identifiziert, von denen 5774 stationär behandelt wurden. Die stärkste Assoziation - auch im heterozygoten Status - zeigte sich hier bei einem SNP im CFTR-Gen, dessen Mutationen für die zystische Fibrose verantwortlich sind. Bei 15.603 Individuen mit afrikanischem Hintergrund wurden 1710 Pneumonien gefunden, 1043 mit Hospitalisierung. Die stärkste Assoziation zeigte sich hier - auch im heterozygoten Status - bei einem SNP im Gen der Hämoglobin$\beta$-Untereinheit, der auch für die Sichelzellanämie verantwortlich ist. Bei beiden Erkrankungen stellt die Pneumonie eine wichtige Komplikation dar. Nach Ausschluss aller Patienten mit zystischer Fibrose und Sichelzellanämie konnte v. a. ein weiterer SNP im Gen R3HCC1L, das für ein Protein unbekannter Funktion kodiert, identifiziert und in 2 unabhängigen Kohorten validiert werden.

In einer GWAS-Analyse zur pneumogenen Sepsis wurden insgesamt $1553 \mathrm{~Pa}$ tienten aus 3 Kohorten untersucht und die Ergebnisse in einer vierten Kohorte validiert. Es zeigte sich, dass Varianten im FER-Gen mit einer deutlich niedrigeren 28-Tage-Mortalität assoziiert waren [14]: Die Mortalität sank von etwa $25 \%$ bei homozygoten Wildtyp-Patienten (TT) auf $15 \%$ bei Trägern einer Kopie des C-Allels und war bei Personen, die homozygot für das C-Allel sind, auf $10 \%$ reduziert. FER kodiert für eine Protein-Tyrosin-Kinase, für die eine Bedeutung in der Leukozytenchemotaxis und der intestinalen Barrierefunktion nachgewiesen wurde.

In einer mexikanischen Studie wurden 91 Patienten mit einer schweren Influenzapneumonie (A/H1N1) und 98 Haushaltskontakte, die dem A/H1N1-Virus ausgesetzt waren und keine Lungenentzündung entwickelten, genotypisiert [15]. Nach Adjustierung für Geschlecht und Komorbiditäten war das Auftreten von SNPs in 4 Genen signifikant erhöht: im Immunglobulin-RezeptorGen FCGR2A, in einem unbekannten Gen auf Chromosom 3 (rs9856661), im RPA-interagierenden Protein RPAIN und in $C 1 Q B P$ aus dem Komplementsystem.

Da Pneumonien im Kindesalter als Risikofaktoren für die Entwicklung einer COPD („chronic obstructive pulmonary disease“) diskutiert werden, wurde bei Patienten aus der COPDGene-Studie nach genetischen Assoziationen mit 
anamnestischen Pneumonien im Kindesalter (843 Fälle, 9091 Kontrollen) oder Pneumonien insgesamt (3766 Fälle, 5659 Kontrollen) gesucht [16]. Allerdings zeigten sich hier keine signifikanten Befunde.

Eine große Metaanalyse aus dem Jahr 2019 errechnete signifikante Effekte auf die Suszeptibilität für Pneumokokkenpneumonie für Varianten der Gene MBL2 und $C D 14$ [17]. Untersuchungen von einzelnen SNPs in kleineren Kohorten ergaben Assoziationen von $H M G B 1$ mit der Pneumoniesuszeptibilität und -schwere [18], von IRF5 mit der Pneumoniesuszeptibilität [19] und von MBL2 und FCN2 mit der Suszeptibilität für Pneumonien mit intrazellulären Erregern [20].

Da manche Genvarianten ihre phänotypische Wirkung nur unter spezifischen Aktivierungsbedingungen zeigen, wurden die rein genetischen Studien durch sog. eQTL-Studien ergänzt, bei denen Genregionen mit Relevanz für die quantitative Ausprägung der entzündlichen Genexpression unter Stimulation z. B. mit bakteriellem Endotoxin ermittelt wurden [21].

Die meisten Untersuchungen adressierten bisher v. a. proteinkodierende $\mathrm{Ge}$ ne. Eine GWAS-Studie an 5000 kenianischen Kindern identifizierte eine Assoziation einer Pneumokokkenbakteriämie mit einer langen nicht proteinkodierenden RNA (Ribonukleinsäure), die nur in neutrophilen Granulozyten exprimiert ist [22]. Im Rahmen weiterer Sequenzierungen des gesamten Genoms und besserer Genannotationen ist mit der Identifizierung weiterer Risiko-SNPs in solchen Genen zu rechnen.

\section{COVID-19}

Seit Dezember 2019 wurden durch die COVID-19-Pandemie über 100 Mio. Menschen von SARS-CoV-2 infiziert und weltweit Regierungen, Gesundheitssysteme und Gesellschaften in ungekannter Weise medizinisch, sozial und wirtschaftlich gefordert. Wesentliche Herausforderungen sowohl für das öffentliche Gesundheitswesen als auch die individuelle medizinische Versorgung bestehen darin, dass zum einen bereits asymptomatische Patienten an-

Pneumologe 2021 · 18:212-217 https://doi.org/10.1007/s10405-021-00385-2

(c) Springer Medizin Verlag GmbH, ein Teil von Springer Nature 2021

\section{W. Bertrams · A. L. Jung $\cdot$ M. Maxheim $\cdot$ B. Schmeck}

\section{Bedeutung der Genetik für die Pneumonie und COVID-19}

\section{Zusammenfassung}

Die Pneumonie ist die Infektionskrankheit mit der weltweit höchsten Mortalität. Die häufigsten Erreger sind Bakterien, es gibt jedoch auch epidemisch oder pandemisch auftretende virale Lungenentzündungen durch Influenza- oder Coronaviren, wie die aktuelle Pandemie durch das SARS Coronavirus 3766 Fälle (SARS-CoV-2). Wichtige Herausforderungen liegen neben dem Auftreten von Antibiotikaresistenzen und Immunpathologien etwa in der Sepsis in der Betrachtung der Suszeptibilität individueller Patienten: Hier werden vor allen Dingen das Lebensalter, Medikamente und Komorbiditäten betrachtet. Es gibt jedoch auch klare Hinweise für genetische Einflüsse auf das individuelle Risiko, an einer Pneumonie zu erkranken oder einen schweren Verlauf der Erkrankung zu entwickeln. In diesem Beitrag wollen wir die genetischen Einflüsse auf die Pneumonie und ihre klinische Bedeutung darstellen.

\section{Schlüsselwörter}

Suszeptibilität · Immundefekt · Polygenetisches Risiko · Mortalität · SARSCoV-2

\section{Role of genetic factors in pneumonia and COVID-19}

\begin{abstract}
Pneumonia causes the highest mortality of all infectious diseases worldwide. The most common pathogens are bacteria but there are also epidemic or pandemic lung infections caused by influenza or coronaviruses, such as the current pandemic caused by the severe acute respiratory syndrome coronavirus 2 (SARS-CoV-2). In addition to the occurrence of antibiotic resistance and immune pathologies, such as in sepsis, important challenges lie in considering the susceptibility of individual patients.
\end{abstract}

Here, age, medication and comorbidities are considered; however, there is also clear evidence of genetic influences on the individual risk of developing pneumonia or developing a severe course of the disease. This article discusses the genetic influences on pneumonia and the clinical significance.

Keywords

Susceptibility · Immune defect · Polygenetic risk - Mortality - SARS-CoV-2 steckend sein können und dass zum anderen die klinische Ausprägung der Infektion ein sehr weites Spektrum aufweist: Die allermeisten Patienten bleiben entweder asymptomatisch oder entwickeln nur leichte Beschwerden, die gut ambulant betreut werden können [23]. Ein kleiner Teil der Patienten entwickelt jedoch im Verlauf eine Pneumonie, die eine Krankenhausaufnahme und ggf. eine intensivmedizinische Betreuung wegen eines ARDS („acute respiratory distress syndrome") und weiterer Organdysfunktionen erfordert. Unter diesen Patienten gibt es nochmals eine kleinere Gruppe, die ein schwerstes systemisches Entzündungssyndrom entwickelt.

Das Alter der Infizierten ist der stärkste bisher identifizierte Risikofaktor: So war das Risiko für einen schweren Krank- heitsverlauf bei einem Lebensalter über 80 Jahren im Vergleich zu einem Lebensalter unter 50 Jahren nach Adjustierung für Begleiterkrankungen 5-fach erhöht, das Sterberisiko sogar 10-fach erhöht [24]. Bei Männern fand sich zudem ein etwa 1,3-fach erhöhtes Sterberisiko. Die Wahrscheinlichkeit für einen schweren oder tödlichen Verlauf war zudem durch das Vorliegen von Übergewicht (BMI [Body-Mass-Index] > 35) sowie chronischen Herz- oder Lungenerkrankungen gering erhöht [23].

Eine Assoziation der Krankheitsschwere mit den AB0-Blutgruppeneigenschaften konnte in einer großen $\mathrm{Me}$ taanalyse mit 7503 COVID-19-Fällen und über 2,9 Mio. Kontrollen nachvollzogen werden: Hier hatten hospitalisierte COVID-19-Patienten wahrscheinli- 
Hier steht eine Anzeige.

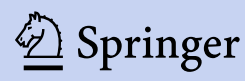


cher Blutgruppe A (Odds Ratio 1,23) und weniger wahrscheinlich Blutgruppe 0 (Odds Ratio 0,77) als die Kontrollen [25]. In GWAS-Studien konnten 4 Assoziationen mit einem schweren Krankheitsverlauf identifiziert werden (• Tab. 1): Das Cluster mit dem stärksten Risiko liegt auf Chromosom 3 und zeigt je nach geografischer Region eine Häufigkeit von bis zu $28 \%$. Es beinhaltet unter anderem SLC6A20, das für einen zellmembranständigen Transporter kodiert, der funktionell mit dem SARSCoV-2-Rezeptor ACE2 interagiert. Weiterhin finden sich dort die Gene für die Zytokinrezeptoren CXCR6 und CCR9, die auf T-Lymphozyten exprimiert werden [26]. Weitere Cluster enthalten Gene OAS1, OAS2 und OAS3, die für interferonabhängige, antivirale Proteine kodieren, sowie das Gen IFNAR2 für einen Interferonrezeptor [27].

\section{》) In GWAS-Studien konnten 4 Assoziationen mit einem schweren Krankheitsverlauf identifiziert werden}

In einem Kandidatengenansatz wurde 659 Patienten mit lebensbedrohlicher COVID-19-Pneumonie und 534 Patienten mit mildem Verlauf auf Mutationen in Genen untersucht, deren Bedeutung für schwere Influenzainfektionen oder andere Virus-assoziierte Phänotypen bereits bekannt war [23]. Hier zeigte sich bei $3,5 \%$ der schweren Verläu$\mathrm{fe}$ entweder eine autosomal-rezessive (IRF7, IFNAR1) oder autosomal-dominante Defizienz (TLR3, UNC93B1, TICAM1, TBK1, IRF3, IRF7, IFNAR1, IFNAR2). Da keiner dieser Patienten zuvor wegen lebensbedrohlicher viraler Infektionen hospitalisiert war, scheinen diese Mutationen eine unerwartet niedrige Penetranz aufzuweisen. Gemeinsam mit den experimentellen Befunden, dass Mutationen von TLR3, IRF7 und IFNAR1 eine funktionelle Bedeutung für SARSCoV-2-Infektionen haben, eröffnet dies zumindest die Möglichkeit, dass $\mathrm{Mu}-$ tationen im Interferon-Signalweg eine signifikante Bedeutung für das Auftreten schwerer COVID-19-Verläufe haben.

\section{Fazit für die Praxis}

- Neben Erreger- und Umweltfaktoren bestimmen auch genetische Eigenschaften die individuelle Suszeptibilität für Infektionskrankheiten, speziell Infektionen der unteren Atemwege.

- Wegen einer unvollständigen Penetranz der Mutationen können auch bei erwachsenen Patienten noch monogenetische Immundefekte identifiziert werden.

- Genetische Testungen werden gegenwärtig nicht routinemäßig angewandt, können im Einzelfall aber sinnvoll sein.

- Bei Patienten mit genetisch bedingt hoher Infektanfälligkeit sollte besonders auf einen vollständigen Impfstatus geachtet werden.

\section{Korrespondenzadresse

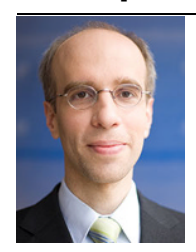 \\ Prof. Dr. Bernd Schmeck Institut für Lungenforschung, Universities of Gießen and Marburg Lung Center (UGMLC), Philipps-Universität Marburg Hans-Meerwein-Str. 2, 35043 Marburg, Deutschland bernd.schmeck@uni- marburg.de}

Prof. Dr. Bernd Schmeck ist Mitglied des Deutschen Zentrums für Lungenforschung, Standort UGMLC, und des Deutschen Zentrums für Infektionsforschung, Standort Marburg-Gießen-Langen

Danksagung. Die Autoren danken Frau Dr. Sabrina von Einem für die Hilfe beim Erstellen des Manuskriptes.

\section{Einhaltung ethischer Richtlinien}

Interessenkonflikt. W. Bertrams, A.L. Jung, M. Maxheim und B. Schmeck geben an, dass kein Interessenkonflikt besteht.

Für diesen Beitrag wurden von den Autoren keine Studien an Menschen oder Tieren durchgeführt. Für die aufgeführten Studien gelten die jeweils dort angegebenen ethischen Richtlinien.

\section{Literatur}

1. Diseases GBD, Injuries C (2020) Global burden of 369 diseases and injuries in 204 countries and territories, 1990 - 2019: a systematic analysis for the Global Burden of Disease Study 2019. Lancet 396(10258):1204-1222
2. Jones B, Waterer G (2020) Advances in community-acquired pneumonia. Ther Adv Infect Dis 7:2049936120969607

3. coronavirus.jhu.edu. Zugegriffen:30. Jan. 2021

4. Casanova JL, Abel L (2021) Lethal infectious diseases as inborn errors of immunity: toward a synthesis of the germ and genetic theories. Annu Rev Pathol 16:23-50

5. Sorensen TI, Nielsen GG, Andersen PK, Teasdale TW (1988) Genetic and environmental influences on premature death in adult adoptees. N Engl J Med 318(12):727-732

6. Mozzi A, Pontremoli C, Sironi M (2018) Genetic susceptibility to infectious diseases: current status and future perspectives from genome-wide approaches. Infect Genet Evol 66:286-307

7. Chapman SJ, Hill AV (2012) Human genetic susceptibility to infectious disease. Nat Rev Genet 13(3):175-188

8. Hawn TR, Verbon A, Lettinga KD, Zhao LP, Li SS, Laws RJ et al (2003) A common dominant TLR5 stop codon polymorphism abolishes flagellin signaling and is associated with susceptibility to legionnaires' disease. JExp Med 198(10):1563-1572

9. Misch EA, Verbon A, Prins JM, Skerrett SJ, Hawn TR (2013) A TLR6 polymorphism is associated with increased risk of Legionnaires' disease. Genes Immun 14(7):420-426

10. Ruiz-Moreno JS, Hamann L, Shah JA, Verbon A, Mockenhaupt FP, Puzianowska-Kuznicka $M$ et al (2018) The common HAQ STING variant impairs cGAS-dependent antibacterial responses and is associated with susceptibility to Legionnaires' disease in humans. PLoS Pathog 14(1):e1006829

11. Shah JA, Emery R, Lee B, Venkatasubramanian $S$, Simmons JD, Brown M et al (2019) TOLLIP deficiency is associated with increased resistance to Legionella pneumophila pneumonia. Mucosal Immunol 12(6):1382-1390

12. Kerner G, Ramirez-Alejo N, Seeleuthner Y, Yang R, Ogishi M, Cobat A et al (2019) Homozygosity for TYK2 P1104A underlies tuberculosis in about 1\% of patients in a cohort of European ancestry. Proc Natl Acad Sci USA 116(21):10430-10434

13. Chen HH, Shaw DM, Petty LE, GraffM, Bohlender RJ, Polikowsky HG et al (2021) Host genetic effects in pneumonia. Am JHum Genet 108(1):194-201

14. Rautanen A, Mills TC, Gordon AC, Hutton P, Steffens M, Nuamah R et al (2015) Genome-wide association study of survival from sepsis due to pneumonia: an observational cohort study. Lancet RespirMed 3(1):53-60

15. Zuniga J, Buendia-Roldan I, Zhao Y, Jimenez L, Torres D, Romo J et al (2012) Genetic variants associated with severe pneumonia in $\mathrm{A} / \mathrm{H} 1 \mathrm{~N} 1$ influenza infection. Eur Respir J 39(3):604-610

16. Hayden LP, Cho MH, McDonald MN, Crapo JD, Beaty TH, Silverman EK et al (2017) Susceptibility to childhood pneumonia: a genome-wide analysis. Am J Respir Cell Mol Biol 56(1):20-28

17. Kloek AT, Brouwer MC, van de Beek D (2019) Host genetic variability and pneumococcal disease: a systematic review and meta-analysis. BMC Med Genomics 12(1):130

18. Song W, Tan H, Wang S, Zhang Y, Ding Y (2019) Association of high mobility group box protein B1 gene polymorphisms with pneumonia susceptibility and severity. Genet Test Mol Biomarkers 23(1):3-11

19. Wang X, Guo J, Wang Y, Xiao Y, Wang L, Hua S (2018) Genetic variants of interferon regulatory factor 5 associated with the risk of community-acquired pneumonia.Gene 679:73-80 


\section{In eigener Sache}

20. van Kempen $G$, Meijvis $\mathrm{S}$, Endeman $\mathrm{H}$, Vlaminckx B, Meek B, de Jong B et al (2017) Mannosebinding lectin and I-ficolin polymorphisms in patients with community-acquired pneumonia caused by intracellular pathogens. Immunology 151(1):81-88

21. Kim S, Becker J, Bechheim M, Kaiser V, Noursadeghi $\mathrm{M}$, Fricker $\mathrm{N}$ et al (2014) Characterizing the genetic basis of innate immune response in TLR4activated human monocytes. Nat Commun 5:5236

22. Kenyan Bacteraemia Study G, Wellcome Trust Case Control C, Rautanen A, Pirinen M, Mills TC, Rockett KA et al (2016) Polymorphism in a lincRNA associates with a doubled risk of pneumococcal bacteremia in Kenyan children. Am J Hum Genet 98(6):1092-1100

23. Zhang Q, Bastard P, Bolze A, Jouanguy E, Zhang SY, Effort CHG et al (2020) Life-threatening COVID19: defective Interferons unleash excessive inflammation. Med (NY) 1(1):14-20

24. Ioannou GN, Locke E, Green P, Berry K, O'Hare AM, Shah JA et al (2020) Risk factors for hospitalization, mechanical ventilation, or death among 10131 US veterans with SARS-coV-2 infection. JAMA Netw Open 3(9):e2022310

25. Golinelli D, Boetto E, Maietti E, Fantini MP (2020) The association between $A B O$ blood group and SARS-CoV-2 infection: a meta-analysis. PLoS ONE 15(9): $\mathrm{e} 239508$

26. Severe Covid GG, Ellinghaus D, Degenhardt F, Bujanda L, Buti M, Albillos A et al (2020) Genomewide association study of severe Covid19 with respiratory failure. $N$ Engl J Med 383(16):1522-1534

27. Pairo-Castineira E, Clohisey S, Klaric L, Bretherick AD, Rawlik K, Pasko D et al (2020) Genetic mechanisms of critical illness in Covid-19. Nature. https://doi.org/10.1038/s41586-020-03065-y

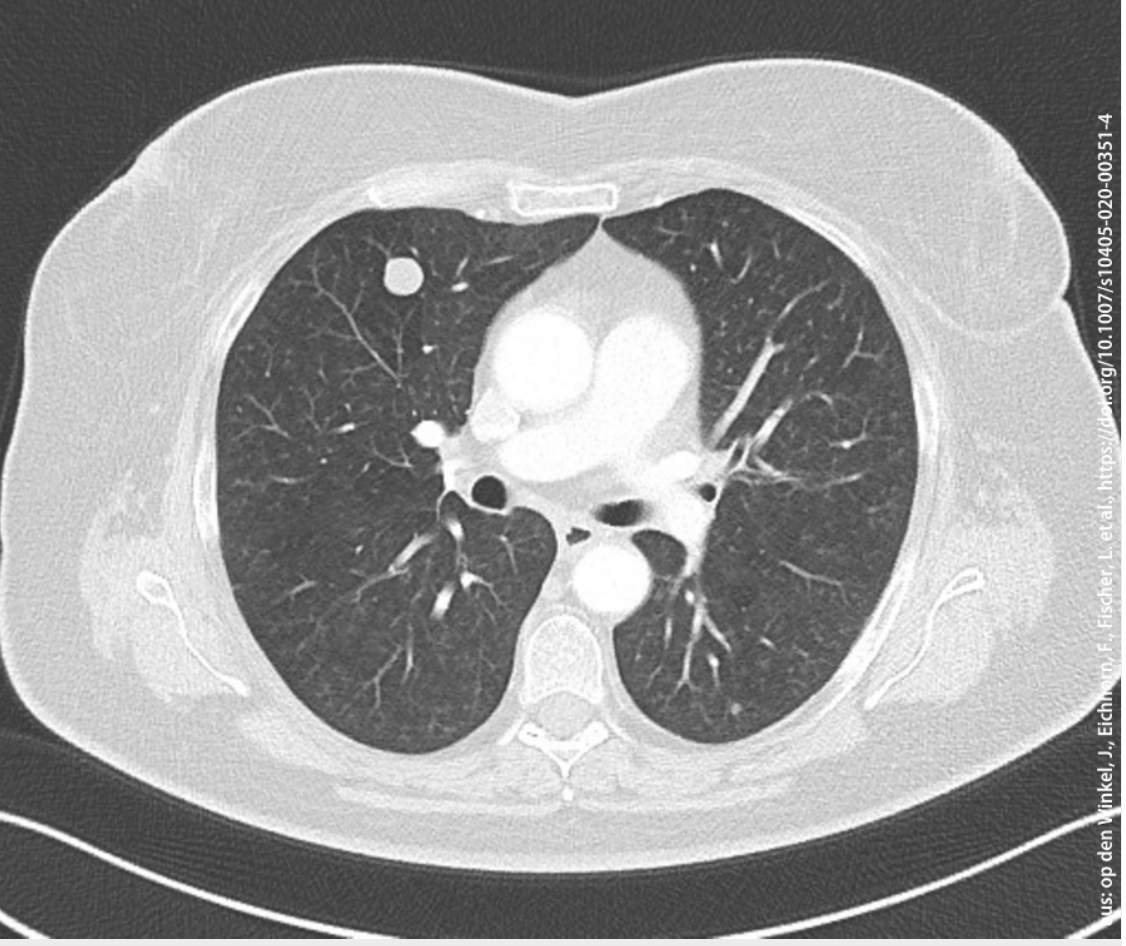

\section{Ihr Fall in Der Pneumologe - reichen Sie Ihr Manuskript für „Bild und Fall" ein}

Sie hatten einen interessanten Fall in Ihrer Praxis? Einen ungewöhnlichen Krankheitsund Behandlungsverlauf? Instruktives Bildmaterial, an dem sich das diagnostische und therapeutische Vorgehen darstellen lässt? Bereiten Sie Ihr Fallbeispiel für die Rubrik „Bild und Fall“ auf und lassen Sie die Leserinnen und Leser von Der Pneumologe an Ihren Erfahrungen teilhaben!

Der Beitrag gliedert sich in zwei Hauptteile:

- Im ersten Teil wird der Fall kurz dargestellt, inklusive Anamnese, klinischem Befund und Diagnostik. Die Leser*innen sollen durch Fallbeschreibung und Bildmaterial alle nötigen Informationen erhalten, um selbst eine Diagnose zu stellen.

- Erst im zweiten Teil wird die Diagnose genannt. Therapie und Verlauf des vorgestellten Falls werden beschrieben; die Leser*innen erhalten weitere Hintergrundinformationen wie z.B. Pathogenese, Differentialdiagnose und weiterführende Diagnostik.
Einen detaillierten Leitfaden finden Sie auf www.DerPneumologe.de

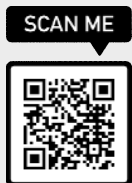

Die Manuskripte werden von zwei unabhängigen Gutachter*innen geprüft. Sie erhalten detaillierte Rückmeldungen und konstruktive Anmerkungen zur Verbesserung Ihres Beitrags.

Die Rubrik wird verantwortet von: Prof. Dr. Daniela Gompelmann, Wien Prof. Dr. F. Joachim Meyer, München Dr. Thomas Wessendorf, Essen

Fragen zur Rubrik beantwortet die Redaktion: Dr. Saskia Rehse

E-Mail: saskia.rehse@springernature.com

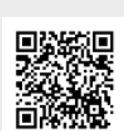

